\title{
Maternal high-fat diet consumption modulates hepatic lipid metabolism and microRNA-122 (miR-122) and microRNA-370 (miR-370) expression in offspring
}

\author{
R. O. Benatti ${ }^{1}$, A. M. Melo ${ }^{1}$, F. O. Borges ${ }^{1}$, L. M. Ignacio-Souza ${ }^{2}$, L. A. P. Simino ${ }^{1}$, M. Milanski ${ }^{1}$, \\ L. A. Velloso ${ }^{3}$, M. A. Torsoni ${ }^{1}$ and A. S. Torsoni ${ }^{1 *}$ \\ ${ }^{1}$ Faculdade de Ciências Aplicadas, Universidade Estadual de Campinas-UNICAMP, Rua Pedro Zaccaria, \\ 1300 Jardim Santa Luiza, Limeira, São Paulo, CEP 13484350, Brazil \\ ${ }^{2}$ Faculdade de Nutrição, Universidade Federal do Mato Grosso-UFMT, Cuiabá, Mato Grosso, Brazil \\ ${ }^{3}$ Departamento de Medicina Interna, Faculdade de Ciências Médicas, Universidade Estadual de Campinas-UNICAMP, \\ Campinas, São Paulo, Brazil
}

(Submitted 29 August 2013 - Final revision received 29 January 2014 - Accepted 31 January 2014 - First published online 25 March 2014)

\begin{abstract}
Maternal consumption of a high-fat diet (HFD) during pregnancy and lactation is closely related to hepatic lipid accumulation, insulin resistance and increased serum cytokine levels in offspring and into their adulthood. MicroRNA (miRNA) have been implicated in cholesterol biosynthesis and fatty acid metabolism. We evaluated the modulation of hepatic fatty acid synthesis (de novo), $\beta$-oxidation pathways, and miRNA-122 (miR-122) and miRNA-370 (miR-370) expression in recently weaned offspring (day 28) of mouse dams fed a HFD (HFD-O) or a standard chow (SC-O) during pregnancy and lactation. Compared with SC-O mice, HFD-O mice weighed more, had a larger adipose tissue mass and were more intolerant to glucose and insulin $(P<0.05)$. HFD-O mice also presented more levels of serum cholesterol, TAG, NEFA and hepatic IкB kinase and c-Jun N-terminal kinase phosphorylation compared with SC-O mice $(P<0 \cdot 05)$. Protein levels of fatty acid synthase, acetyl-CoA carboxylase and 3-hydroxy-3-methylglutaryl-CoA reductase were similar in HFD-O and SC-O mice, whereas expression levels of SCD1 mRNA and protein were more abundant in HFD-O mice than in SC-O mice $(P<0 \cdot 05)$. Interestingly, mRNA expression levels of the $\beta$-oxidation-related genes $A C A D V L$ and $C P T 1$ were decreased in HFD-O mice $(P<0 \cdot 05)$. Furthermore, the expression of miR-122 was reduced but that of miR-370 was increased in HFD-O mice compared with that in SC-O mice $(P<0.05)$. Changes in hepatic lipid metabolism were accompanied by increased mRNA content of AGPAT1 and TAG deposition in HFD-O mice $(P<0 \cdot 05)$. Taken together, the present results strongly suggest that maternal consumption of a HFD affects the early lipid metabolism of offspring by modulating the expression of hepatic $\beta$-oxidation-related genes and miRNA that can contribute to metabolic disturbances in adult life.
\end{abstract}

\section{Key words: Lipid metabolism: Maternal imprinting: Obese mice: High-fat diet: MicroRNA}

Maternal obesity predisposes offspring to metabolic disorders. Nutritional overload during early or prenatal life can cause permanent damage to offspring ${ }^{(1-3)}$. Consumption of a highfat diet (HFD) during pregnancy leads to the activation of macrophages in the maternal placenta that culminates in an inflammatory environment for fetal development ${ }^{(4)}$. Maternal body-weight gain during critical phases of offspring development results in greater adiposity, body weight, liver TAG content and hepatic steatosis in adult life ${ }^{(5,6)}$. Furthermore, the uterus and blastocysts from obese dams exhibit inflammatory signals linking maternal obesity to increased predisposition of offspring to obesity later in life $\mathrm{e}^{(7)}$.
Liver steatosis is characteristic of obesity and diabetes and is closely associated with inflammatory signals ${ }^{(8,9)}$. Maternal consumption of a HFD during pregnancy has been shown to result in increased fetal hepatic lipid accumulation, oxidative stress and apoptosis in a non-human primate model of maternal obesity ${ }^{(10)}$. Recently, we observed that offspring of obese dams displayed increased fatty liver deposition immediately after weaning and into their adulthood ${ }^{(6)}$. In addition, Heerwagen et al. ${ }^{(11)}$ showed that, at embryonic day 18.5, fetuses of obese dams had higher fetal liver TAG deposition than those of lean dams. The metabolic pathways leading to the development of hepatic steatosis are multiple, including enhanced NEFA release from

Abbreviations: $A C A D V L$, acyl-CoA dehydrogenase, very long chain; ACC, acetyl-CoA carboxylase; $A G P A T 1,1$-acylglycerol-3-phosphate $O$-acyltransferase 1; $A M P K$, AMP-activated protein kinase; HFD, high-fat diet; HFD-O, offspring of dams fed a high-fat diet; HMGCR, 3-hydroxy-3-methylglutaryl-CoA reductase; JNK, c-Jun N-terminal kinase; miR-122, miRNA-122; miR-370, miRNA-370; miRNA, microRNA; SC, standard chow; SC-O, offspring of dams fed a standard chow; SCD1, stearoyl-CoA desaturase 1.

*Corresponding author: A. S. Torsoni, fax +55 193701 6680, email adriana.torsoni@fca.unicamp.br 
Table 1. Nutritional composition of the experimental and standard chow diets fed to mice during gestation and lactation

\begin{tabular}{lcc}
\hline & Chow diet $^{*}$ & High-fat diet \\
\hline Net protein (g \%) & 20.0 & 23.3 \\
Diethyl ether extract (fat content, g \%) & 4.0 & 24.0 \\
Carbohydrates (g \%) & 65.95 & 42.1 \\
Fibrous matter (g \%) & 5.0 & 5.55 \\
Mineral mix (g \%) & 3.5 & 3.5 \\
Vitamin mix (g \%) & 1.0 & 1.0 \\
Choline & 0.25 & 0.25 \\
Cystine & 0.3 & 0.3 \\
Total & 100.0 & 100.0 \\
Energy (kJ/g) & 14.6 & 19.3 \\
\hline
\end{tabular}

${ }^{*}$ NUVILAB ${ }^{\circledast} \mathrm{Cr}-1$; Nuvital.

adipose tissue (lipolysis), increased de novo fatty acid synthesis (lipogenesis) and decreased $\beta$-oxidation activity ${ }^{(12,13)}$

Epigenetic mechanisms have been associated with metabolic imprinting of and damages to adulthood offspring ${ }^{(14,15)}$.

MicroRNA (miRNA) are endogenous non-coding RNA of approximately twenty-two nucleotides in length that regulate various metabolic processes and diseases ${ }^{(16)}$. miRNA-122 (miR-122) is liver-specific and the most abundant miRNA in this organ, accounting for approximately $70 \%$ of the total miRNA population. Functional studies have shown that $m i R-$ 122 is involved in multiple metabolic processes including cholesterol biosynthesis, fatty acid synthesis and oxidation ${ }^{(17)}$. The repression of $m i R-122$ results in hepatic insulin resistance by protein-tyrosine phosphatase $1 \mathrm{~B}$ induction ${ }^{(18)}$, similar to the repression of miRNA-370 ( $m i R-370)$. Iliopoulos et $a l^{(19)}$ showed that $m i R-370$ directly down-regulated the expression of the gene encoding carnitine palmitoyltransferase $1 \alpha$ $(C P T 1 \alpha)$, that controls fatty acid oxidation. Therefore, miRNA may contribute to the induction of metabolic damage associated with fatty liver deposition in the offspring of obese dams. We used offspring mice that were recently weaned from obese dams to investigate the modulation of hepatic fatty acid synthesis (de novo), $\beta$-oxidation pathways, and expression of $m i R-122$ and $m i R-370$ by maternal consumption of a HFD during pregnancy and lactation.

\section{Materials and methods}

\section{Ethics statement}

All experiments were performed in accordance with the guidelines of the Brazilian College for Animal Experimentation (COBEA) and approved by the Committee for Ethics in Animal Experimentation (protocol no. 2864-1) at the State University of Campinas - UNICAMP (Campinas, São Paulo, Brazil).

\section{Animals}

A total of ten virgin female and male Swiss mice (7 weeks old) that were specific pathogen free were obtained from the Animal Breeding Center at the University of Campinas (Campinas, SP, Brazil) for mating. Before mating, the females were randomly fed ad libitum either a HFD or standard laboratory chow (SC) for 2 weeks for adaptation (Table 1) and received filtered water ad libitum. Mating was performed by housing females with adult males (fed SC) for 1 week, and pregnancy was confirmed by the examination of vaginal smears for the presence of sperm.

Pregnant females were maintained in individual polypropylene microisolators with a bed of pinewood autoclaved (about $60 \mathrm{~g}$ ) in a rack at $22 \pm 1^{\circ} \mathrm{C}$ with lights on from 06.00 to 18.00 hours. They received the same diet (HFD or SC) during pregnancy and lactation as before mating. The HFD was prepared according to AIN-93G modified for high fat (45\%) content (Table 1). Offspring were divided into two groups according to maternal feeding: offspring of female mice fed the HFD (HFD-O group) and offspring of female mice fed the SC (SC-O group). At day 1 after birth, the litters of both groups (HFD-O and SC-O) were adjusted to eight pups each to ensure a standard litter size per mother. The pups were weaned on day 18 and separated according to sex. Male offspring were maintained in the same environmental conditions fed the SC after weaning until the end of the experimental period (day 28), according to the experimental protocol (Fig. 1).

Sample size was estimated from previous experiments and confirmed using a simple power and sample size calculation, available at the website of the Biomathematics Division of the Department of Pediatrics at the College of Physicians and Surgeons at Columbia University ${ }^{(20)}$. The number of individual experiments was representative of at least three different litters.

The total number of animals used in each experiment is indicated in the figure legends.

\section{Biochemical analysis}

At the end of the experimental period (day 28) and after overnight fasting, all mice were killed and blood samples were collected and centrifuged, and serum aliquots were used to measure the levels of serum TAG (glycerol-3-phosphate oxidase-phenol + aminophenazone (GPO-PAP); Roche Diagnostics), cholesterol (cholesterol oxidase-phenol + aminophenazone (CHOD-PAP); Roche Diagnostics) and NEFA (acyl-CoA synthase-acyl-CoA oxidase (ACS-ACOD); Wako Chemicals) by enzymatic colorimetry.

Frozen tissues $(200 \mathrm{mg}$ ) from SC-O and HFD-O specimens were homogenised in $1.5 \mathrm{ml}$ of $\mathrm{PBS}$ and processed as described by others ${ }^{(21,22)}$. The protein concentration of the homogenate was determined, and an aliquot of $300 \mu \mathrm{l}$ was extracted with $5 \mathrm{ml}$ of chloroform-methanol (2:1) and $0.5 \mathrm{ml}$

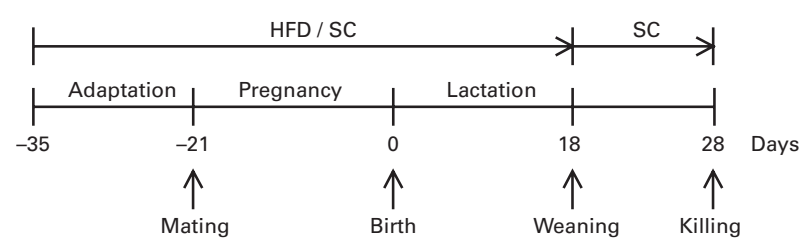

Fig. 1. Experimental protocol followed to obtain offspring of dams fed a highfat diet (HFD; HFD-O mice) and standard chow (SC; SC-O mice). Difference between the groups is based only on the diet offered to dams during the periods of adaptation, pregnancy and lactation. After weaning, HFD-O and $\mathrm{SC}-\mathrm{O}$ mice were fed the SC diet. 
of $0 \cdot 1 \% \mathrm{H}_{2} \mathrm{SO}_{4}(\mathrm{v} / \mathrm{v})$. An aliquot of the organic phase was collected, dried under $\mathrm{N}_{2}$ and resuspended in $2 \%$ Triton X-100. TAG content was determined using a commercially available kit (GPO-PAP; Roche Diagnostics).

\section{Intraperitoneal glucose tolerance test and intraperitoneal insulin tolerance test}

For intraperitoneal glucose tolerance test, offspring of HFDand SC-fed mice at day 28 were starved for $12 \mathrm{~h}$, fed for $2 \mathrm{~h}$ and starved for an additional $4 \mathrm{~h}$ before intraperitoneal injection of glucose ( $1 \mathrm{~g} / \mathrm{kg}$ of a $25 \%$ solution of D-glucose) as described by Bonora $e t a l .{ }^{(23)}$. Blood samples were collected from the tail at $0,10,15,30,60$ and $120 \mathrm{~min}$ after injection for the measurement of blood glucose concentration. The AUC of glycaemia $v$. time was calculated above each individual baseline (basal glycaemia) to estimate glucose tolerance. The AUC of glycaemia $(\mathrm{mmol} / \mathrm{l}) v$. time $(120 \mathrm{~min})$ was calculated above each individual baseline (basal glycaemia) to estimate glucose tolerance, using the trapezoidal method.

For intraperitoneal insulin tolerance test, insulin $(6.00 \mathrm{nmol}$ (1.0 IU) $/ \mathrm{kg}$ body weight) was administered by intraperitoneal injection, and tail blood samples were collected at 0, 3, 6, 9 and $12 \mathrm{~min}$ after insulin administration to mice (day 28), following the same fasting protocol. The constant for the glucose disappearance rate during the test ${ }^{(24)}$ was calculated using the formula $0.693 / t_{1 / 2}{ }^{(23)}$.

For both glucose tolerance test and insulin tolerance test, glycaemia was determined on an Accu-Chek Performa glucometer (Roche Diagnostics).

\section{Food intake measurement}

Food intake was estimated during $24 \mathrm{~h}$ over a period of four alternate days after weaning. The average was considered as food intake $(\mathrm{g} / \mathrm{d})$.

\section{Quantitative real-time PCR}

Total hepatic RNA was extracted using TRIzol reagent (Life Technologies Corporation), according to the manufacturer's recommendations. Total RNA was quantified on a NanoDrop ND- 2000 (Thermo Electron) and its integrity verified by agarose gel electrophoresis. Reverse transcription was performed with $3 \mu \mathrm{g}$ of total RNA and a High-Capacity cDNA Reverse Transcription kit (Life Technologies Corporation). Relative expression was determined using the Taqman detection system and primers for the target genes: Mm 00772290_m1 stearoyl-coenzyme A desaturase 1 (SCD1); Mm 01282499_m1 3-hydroxy-3-methylglutaryl-CoA reductase (HMGCR); Mm 01304277_m1 acetylCoA carboxylase $\alpha$ (ACACA); Mm 00662313_m1 fatty acid synthase (FASN); Mm 00479699_g1 1-acylglycerol-3-phosphate O-acyltransferase 1 (AGPAT1); Mm 01247712_m1 hepatocyte nuclear factor 4, $\alpha$ (HNF4 $\alpha)$; Mm 00444293_m1 acyl-CoA dehydrogenase, very long chain $(A C A D V L)$; Mm 01231183_m1 CPT1 $\alpha$; Mm 01296700_m1 AMP-activated protein kinase $(A M P K)$ (Life Technologies Corporation); glyceraldehyde3-phosphate dehydrogenase $(G A P D H)$ as the endogenous control (4352339E mouse GAPD; Life Technologies Corporation). Each PCR contained $20 \mathrm{ng}$ of complementary DNA.

Gene expression was quantified by real-time PCR performed on an ABI Prism 7500 Fast platform. Data were analysed using the Sequence Detection System 2.0.5 (Life Technologies Corporation) and expressed as relative values determined by the comparative threshold cycle $\left(C_{\mathrm{t}}\right)$ method $\left(2^{-\Delta \Delta C_{t}}\right)$, according to the manufacturer's recommendation.

\section{MicroRNA isolation and quantification}

miRNA was extracted and purified from the liver of SC-O and HFD-O mice using a mirVana miRNA Isolation Kit (Life Technologies Corporation). The relative expression of $m i R-122$ and $m i R-370$ was determined using the TaqMan detection system (Life Technologies Corporation), the appropriate primers (ID 002245 and 002275 , respectively), and U6 spliceosomal RNA (ID 001973) and miR-16 (ID 000391) as endogenous controls (Life Technologies Corporation). Gene expression was quantified as described in the preceding section.

\section{Immunoblotting}

Tissue samples (day 28) were homogenised in freshly prepared ice-cold buffer (1\% (v/v) Triton X-100, 0.1 M-Tris, $\mathrm{pH} 7 \cdot 4,0 \cdot 1 \mathrm{~m}$-sodium pyrophosphate, 0.1 M-sodium fluoride, 0.01 M-EDTA, 0.01 M-sodium vanadate, $0.002 \mathrm{~m}$-phenylmethanesulfonyl fluoride and $0.01 \mathrm{mg}$ aprotinin $/ \mathrm{ml})$. The insoluble material was removed by centrifugation $(10000 \mathrm{~g})$ for $25 \mathrm{~min}$ at $4^{\circ} \mathrm{C}$. The protein concentration of the supernatant was determined by the Bradford dye-binding method. The supernatant was resuspended in Laemmli sample buffer and boiled for

Table 2. Body composition and glycaemia from the control (standard chow (SC))- and high-fat diet (HFD)-fed dams (Mean values with their standard errors)

\begin{tabular}{|c|c|c|c|c|c|c|c|c|c|c|c|c|}
\hline & \multicolumn{3}{|c|}{ G1BW (g) } & \multicolumn{3}{|c|}{ L1BW (g) } & \multicolumn{3}{|c|}{ eWAT (g) } & \multicolumn{2}{|c|}{ Gly $(\mathrm{mmol} / \mathrm{l})$} & \\
\hline & Mean & SEM & $n$ & Mean & SEM & $n$ & Mean & SEM & $n$ & Mean & SEM & \\
\hline SC & $29 \cdot 3$ & 0.4 & 5 & 32.0 & 0.4 & 5 & 0.1 & 0.01 & 5 & 6.97 & 0.5 & \\
\hline HFD & $42 \cdot 8^{\star}$ & 0.9 & 5 & $42 \cdot 5^{\star}$ & 1.6 & 5 & $1.7^{*}$ & 0.2 & 5 & $7 \cdot 13$ & 0.18 & \\
\hline
\end{tabular}

G1BW, body weight at first gestational week; L1BW, body weight at first lactational week; eWAT, epigonadal white adipose tissue at day 28; Gly, fasting glycaemia at day 28.

${ }^{*}$ Mean value was significantly different from that of the SC-fed group $(P \leq 0.05)$. 
Table 3. Body composition and food intake of the offspring of dams fed a standard chow (SC-O group) and high-fat diet (HFD-O group)

(Mean values with their standard errors)

\begin{tabular}{|c|c|c|c|c|c|c|c|c|c|c|c|c|c|c|c|}
\hline & \multicolumn{3}{|c|}{ d1BW (g) } & \multicolumn{3}{|c|}{ d28BW (g) } & \multicolumn{3}{|c|}{ eWAT (g) } & \multicolumn{3}{|c|}{ rWAT $(\mathrm{g})$} & \multicolumn{3}{|c|}{ Food intake $(\mathrm{g} / \mathrm{d})$} \\
\hline & Mean & SEM & $n$ & Mean & SEM & $n$ & Mean & SEM & $n$ & Mean & SEM & $n$ & Mean & SEM & $n$ \\
\hline SC-O & 2.02 & 0.02 & 40 & $16 \cdot 76$ & 0.49 & 24 & 0.06 & 0.008 & 19 & 0.02 & 0.007 & 10 & 6.45 & 0.17 & 5 \\
\hline HFD-O & 2.06 & 0.03 & 32 & $21 \cdot 88^{\star}$ & 0.47 & 22 & $0 \cdot 18^{\star}$ & 0.014 & 21 & $0.04^{\star}$ & 0.004 & 20 & $7 \cdot 01^{\star}$ & 0.41 & 5 \\
\hline
\end{tabular}

d1BW, body weight at first postnatal day; d28BW, body weight at postnatal day 28; eWAT, epididymal white adipose tissue; rWAT, retroperitoneal white adipose tissue.

${ }^{*}$ Mean value was significantly different from that of the SC-O group $(P \leq 0.05)$.

5 min before separation by SDS-PAGE using a miniature slab gel apparatus (Bio-Rad). Electrotransfer of proteins from the gel to nitrocellulose was performed for $90 \mathrm{~min}$ at $120 \mathrm{~V}$ (constant). The nitrocellulose blots were probed with specific antibodies. Antibodies to phospho-c-Jun N-terminal kinase (JNK, SC-1648), phospho-IкB kinase (SC-21660), NF-кBp65 (SC-8008), fatty acid synthase (FAS, SC-20140), HMGCR (SC-33827), CPTI (SC98834), ACADVL (SC-376239) and HNF4 $\alpha$ (SC-8987) were obtained from Santa Cruz Biotechnology, Inc. Antibodies against phospho-acetyl-CoA carboxylase (ACC, 3661S), ACC (3676S), phospho-AMPK (2535S) and AMPK (2532S) were obtained from Cell Signaling Technology, Inc. Antibodies against $\beta$-actin (ab8227) and SCD1 (ab19862) were obtained from Abcam.

After incubation with specific antibodies, the blots were incubated with horseradish peroxidase-conjugated secondary antibodies (KPL). Proteins recognised by the secondary antibodies were detected by chemiluminescence (Amersham ECL kit (RPN 2232)) as visualised by the exposure of the blot to Kodak XAR film. Band intensities were quantified by optical densitometry of developed autoradiographs (Scion Image software; ScionCorp), and the intensities of the bands were normalised to those of total protein or the loading control $\beta$-actin.

\section{Liver histochemistry analysis}

Fragments of liver from the HFD-O and SC-O mice were fixed in $10 \%$ formalin for $24 \mathrm{~h}$. Tissue samples were subsequently processed using routine histological methods and embedded in paraffin. Briefly, tissues were sectioned $(5 \mu \mathrm{m})$, stained with haematoxylin-eosin and analysed by light microscopy (Leica FW 4500 B microscope; Leica Imaging Systems). Samples were coded and assessed by two independent, blinded observers.

\section{Statistical analysis}

All numerical results are expressed as means with their standard errors of the indicated number of experiments. Blot results are presented as direct band comparisons in autoradiographs and quantified by densitometry using Scion Image software (ScionCorp). Student's $t$ tests of unpaired samples were employed for determining a significance level of $P<0 \cdot 05$.

\section{Results}

To examine the effects of maternal HFD-induced obesity on offspring metabolism and miRNA expression, we first characterised our experimental model. Table 2 shows that in early gestational and lactation periods, HFD-fed dams had a higher body weight than SC-fed dams (1.5- and 1.3-fold, respectively). HFD-fed dams also showed higher (17-fold) epigonadal adipose tissue than SC-fed dams. On day 1, the body weights of SC-O and HFD-O mice did not differ significantly. At day 28, HFD-O mice were significantly heavier (approximately 1.3-fold) than SC-O mice. In addition, HFD-O mice had larger epididymal $(3 \cdot 1$-fold $)$ and retroperitoneal $(2 \cdot 0$-fold $)$ fat pads than SC-O mice (Table 3). Furthermore, food intake was little higher in HFD-O (1-1-fold) than in SC-O mice (Table 3). In agreement with these results, maternal consumption of the HFD during pregnancy and lactation resulted in glucose intolerance and insulin resistance in the offspring, as indicated by the AUC during the glucose tolerance test and кITT (intraperitoneal insulin tolerance test constant) calculated from the insulin tolerance test (Table 4), respectively. In addition,

Table 4. Metabolic parameters of the offspring of dams fed a standard chow (SC-O group) and high-fat diet (HFD-O group) at day 28 (Mean values with their standard errors; $n 6-12$ pups per group)

\begin{tabular}{|c|c|c|c|c|c|c|c|c|c|c|c|c|}
\hline & \multicolumn{2}{|c|}{$\mathrm{CHO}(\mathrm{mmol} / \mathrm{l})$} & \multicolumn{2}{|c|}{ NEFA (mmol/l) } & \multicolumn{2}{|c|}{ TAG (mmol/l) } & \multicolumn{2}{|c|}{$\begin{array}{l}\text { HTAG }(\mu \mathrm{g} / \mathrm{mg} \\
\text { tissue) }\end{array}$} & \multicolumn{2}{|c|}{$A \cup C(A U)$} & \multicolumn{2}{|c|}{ кITT (\%/min) } \\
\hline & Mean & SEM & Mean & SEM & Mean & SEM & Mean & SEM & Mean & SEM & Mean & SEM \\
\hline $\mathrm{C}-\mathrm{O}$ & $3 \cdot 10$ & 0.15 & 0.84 & 0.07 & 0.48 & 0.05 & $5 \cdot 20$ & 0.50 & 653 & 0.35 & $12 \cdot 02$ & 4.05 \\
\hline HFD-O & $4.38^{*}$ & 0.17 & $1.43^{*}$ & 0.06 & $1 \cdot 18^{\star}$ & 0.08 & $7 \cdot 80^{*}$ & $0 \cdot 80$ & $830^{*}$ & 0.27 & $7 \cdot 57^{*}$ & 1.72 \\
\hline
\end{tabular}

$\mathrm{CHO}$, serum cholesterol; TAG, serum TAG; HTAG, hepatic TAG; AUC, AUC of serum glucose curve during the glucose tolerance test; AU, arbitrary 
(a)
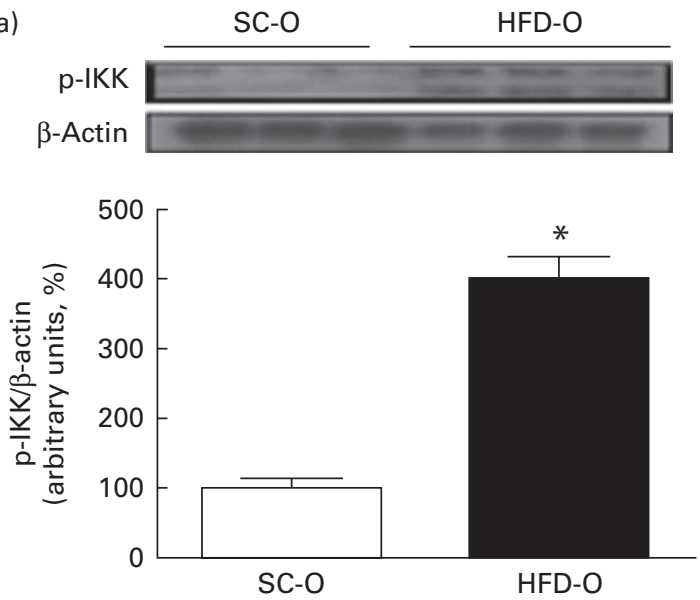

(b)
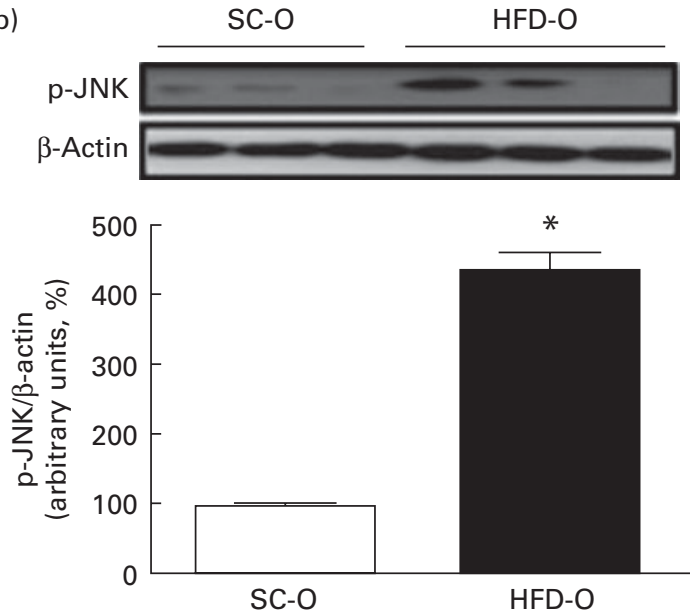

(c)
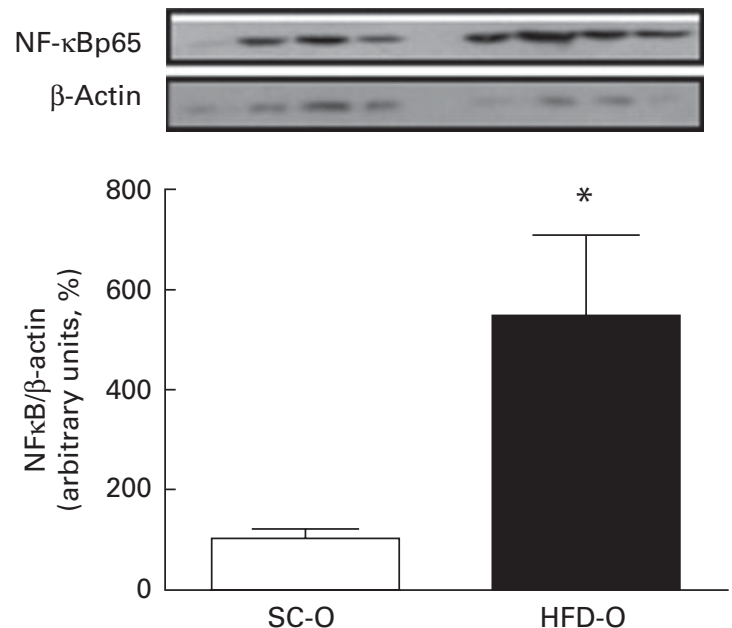

Fig. 2. Western blotting (WB) of hepatic phospho-IKB kinase (p-IKK) (a), phospho-c-Jun N-terminal kinase (p-JNK) (b) and NF- $\mathrm{KB}(\mathrm{NF}-\kappa \mathrm{Kp} 65)$ (c) in the offspring of dams fed a high-fat diet (HFD-O group) and standard chow (SC-O group) (day 28). For the control of gel loading in WB, membranes were reblotted with $\beta$-actin. Values are means $(n-7)$, with their standard errors represented by vertical bars. * Mean value was significantly different from that of the SC-O group $(P \leq 0.05)$.

serum components such as cholesterol, TAG and NEFA were more abundant in HFD-O mice than in SC-O mice (Table 4).

Because HFD consumption leads to peripheral inflammation and defective regulation of energy homeostasis ${ }^{(6)}$, pro-inflammatory pathways in the liver were investigated (day 28). As shown in Fig. 2, hepatic ІкB kinase phosphorylation, JNK phosphorylation and NF-кBp65 were higher in HFD-O mice than in SC-O mice (4.0-, 4.3- and 5.2-fold, respectively; Fig. 2(a)-(c)).

We next investigated the influence of maternal obesity on lipogenic gene expression in the offspring. HFD-O and SC-O mice expressed similar levels of hepatic phospho- $A C C, F A S$ and HMGCR and ACC and FASN mRNA (Fig. 3(a) and (c)-(e)). However, HFD-O mice expressed more hepatic AGPAT1 mRNA (1.7-fold; Fig. 3(e)), in addition to SCD1 protein and mRNA (2.2- and 3.0-fold, respectively) than SC-O mice (Fig. 3(b) and (e)), even though HMGCR mRNA levels were reduced (Fig. 3(e)).
To evaluate the influence of maternal obesity on $\beta$-oxidation-related gene expression in the offspring, $A M P K$ phosphorylation and $A C A D V L$ and $C P T 1$ expression were determined. As shown in Fig. 4(a), hepatic $A M P K$ phosphorylation was similar in SC-O and HFD-O mice (day 28). However, levels of ACADVL protein and mRNA and CPT1 mRNA were lower in HFD-O mice than in SC-O mice (75, 30 and $40 \%$, respectively; Fig. 4(b) and (c)). In contrast, $A M P K$ mRNA levels were higher (1.3-fold) in the liver of HFD-O mice than in that of SC-O mice (Fig. 4(c)). Interestingly, HFD-O mice had an increased hepatic TAG content (1.5-fold) compared with SC-O mice (Table 4). The presence of vacuoles that contained lipids within hepatocytes as shown by haematoxylin-eosin-stained liver sections from the HFD-O mice (Fig. 5(c) and (d)) corroborates molecular results for this group, while the SC-O group had a normal liver structure (Fig. 5(a) and (b)).

Compared with SC-O mice, HFD-O mice expressed similar levels of hepatic HNF $4 \alpha$ protein and mRNA (Fig. 6(a) and (b)), 
(a)
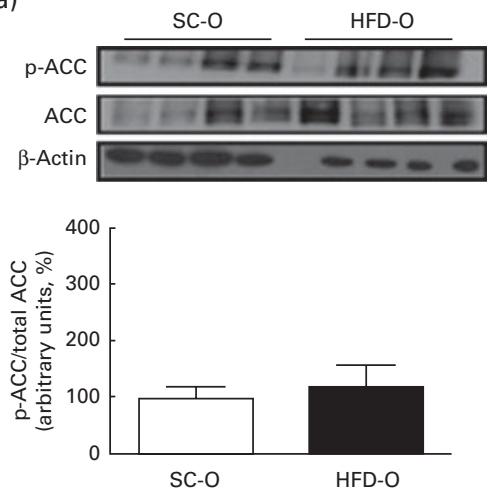

(b)
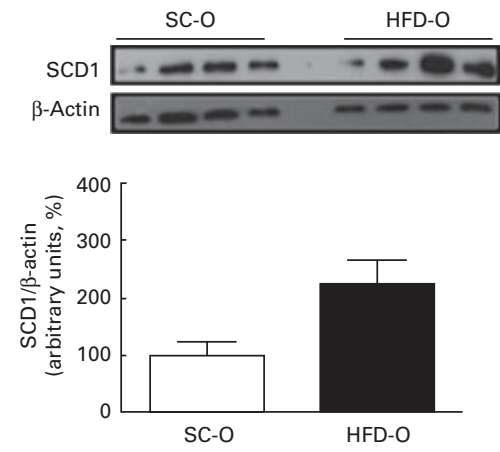

(c)
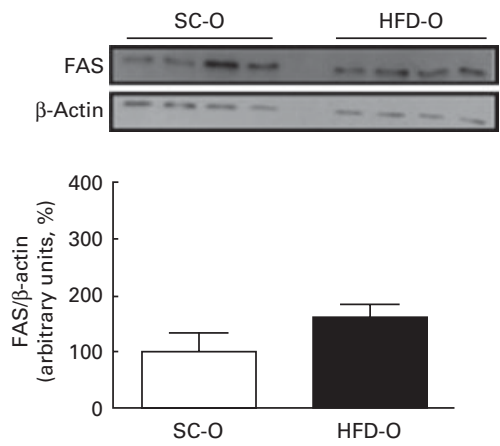

(d)
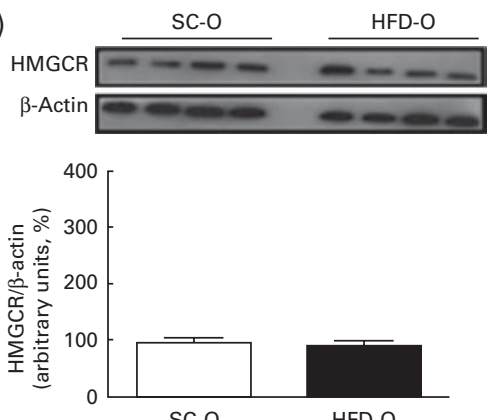

(e)

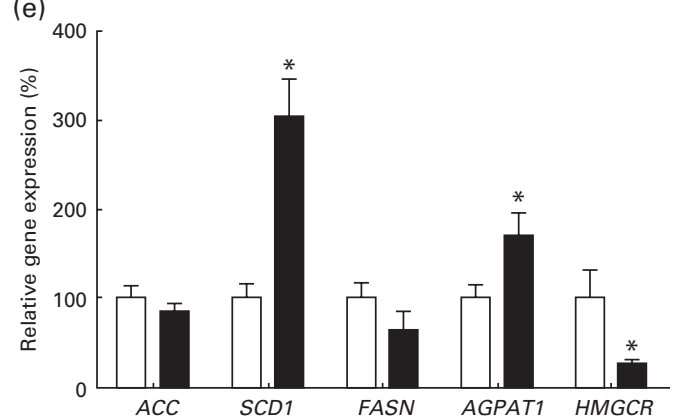

Fig. 3. Western blotting (WB) of hepatic phospho-acetyl-CoA carboxylase (p-ACC)/ACC (a), stearoyl-CoA desaturase 1 (SCD1) (b), fatty acid synthase (FAS) (c) and 3-hydroxy-3-methylglutaryl-CoA reductase (HMGCR) (d) in the offspring of dams fed a high-fat diet (HFD-O group, $\square$ ) and standard chow (SC-O group, $\square$ ) (day 28). For the control of gel loading in WB, membranes were reblotted with $\beta$-actin. mRNA levels (quantitative real-time PCR (qRT-PCR)) of hepatic ACC, $S C D 1$, fatty acid synthase (FASN), 1-acylglycerol-3-phosphate O-acyltransferase 1 (AGPAT1) and HMGCR (e) in mice (day 28). For relative gene expression analysis, glyceraldehyde-3-phosphate dehydrogenase was used as the endogenous control. Values are means ( $n 4$ for WB and $n 8-12$ for qRT-PCR), with their standard errors represented by vertical bars. * Mean value was significantly different from that of the SC-O group $(P \leq 0.05)$.

reduced (25\%) levels of hepatic miR-122 (Fig. 6(c)) and increased (3-fold) levels of $m i R-370$ (Fig. 6(d)).

\section{Discussion}

It has been demonstrated that high-fat diet consumption activates pro-inflammatory pathways, causes endoplasmic reticulum stress, ectopic lipid deposition and insulin resistance, and contributes to other co-morbidities associated with obesity $^{(25-31)}$. Moreover, maternal consumption of a HFD during pregnancy and lactation has also been related to metabolic disturbances in adult offspring ${ }^{(6,7,10,32)}$.

Hepatic damage associated with metabolic changes promoted by maternal consumption of a HFD during pregnancy and lactation has been described in offspring at different periods of development ${ }^{(6,10,11,13)}$. Fatty liver is characteristic of obesity and diabetes and is closely associated with inflammatory signals ${ }^{(8,9)}$. As shown here, HFD-O mice weighed more, had a larger adipose tissue mass than SC-O mice and were more glucose- and insulin-intolerant. Furthermore, HFD-O mice expressed more hepatic NF-кBp65, phosphoJNK and phospho-IкB kinase than SC-O mice, suggesting that liver insulin resistance may be associated with the activation of pro-inflammatory pathways, as described previously ${ }^{(28,33,34)}$. However, recent conflicting findings question the importance of hepatic inflammation in the development of insulin resistance. Wiedemann et al. ${ }^{(35)}$ showed that adipose tissue inflammation contributed to HFD-induced hepatic insulin resistance, whereas Turner et $a l .{ }^{(36)}$ found that consumption of a HFD for 3-4 weeks induced insulin resistance without the evidence of inflammation in the liver, adipose tissue or skeletal muscle. Moreover, they detected adipose tissue inflammation only after 16 weeks of HFD consumption. Interestingly, TAG content and the amount of a diacylglycerol species were increased in the liver after 1 week of HFD consumption, whereas changes in ceramide abundance occurred only after the development of insulin resistance ${ }^{(36)}$. Thus, ectopic lipid accumulation appears to correlate with insulin resistance ${ }^{(37,38)}$, but the lipid classes that mediate insulin resistance are unknown ${ }^{(39)}$. We did not evaluate inflammatory markers in the adipose tissue, but we demonstrated increased adipose tissue mass and hepatic lipid accumulation in HFD-O mice. Furthermore, it is important to point out that although HFD can impair the effect of insulin on glucose production, insulin-stimulated lipid synthesis was not altered in the present study, indicating selective insulin resistance ${ }^{(40)}$. Thus, damage to hepatic glucose homeostasis can occur in the offspring of HFD-fed dams in the absence of changes in lipid synthesis. Interestingly, in a previous study employing the same model as used here, we demonstrated that HFD-O mice (day 82) exhibited liver insulin resistance and JNK and 
(a)
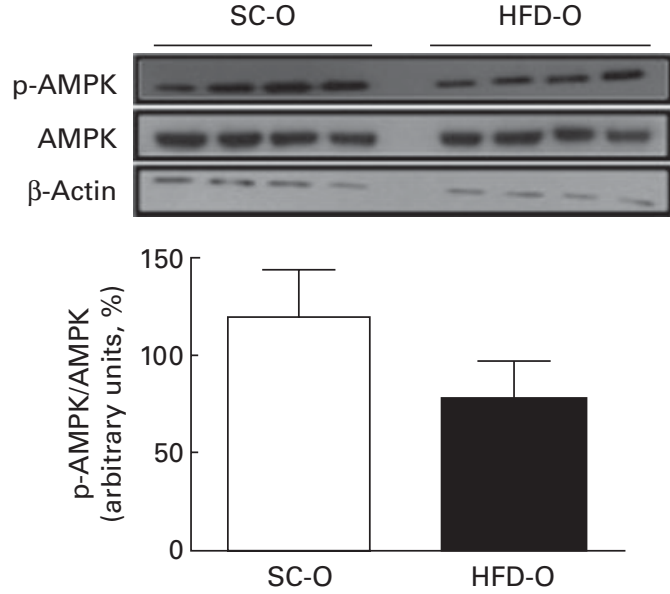

(b)
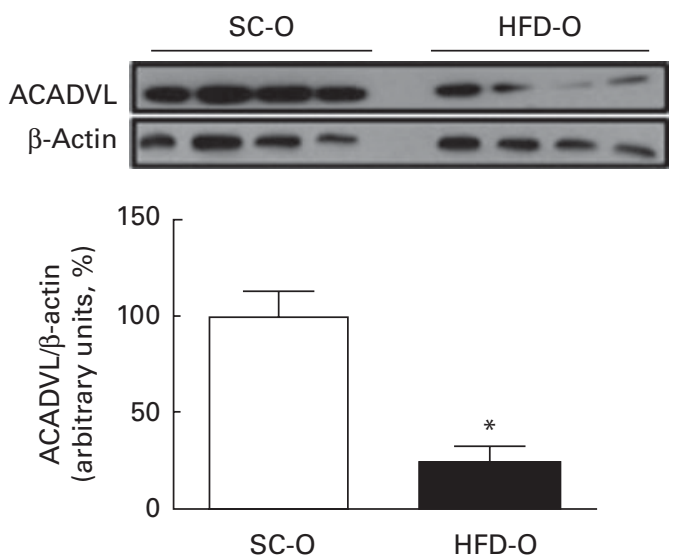

(c)

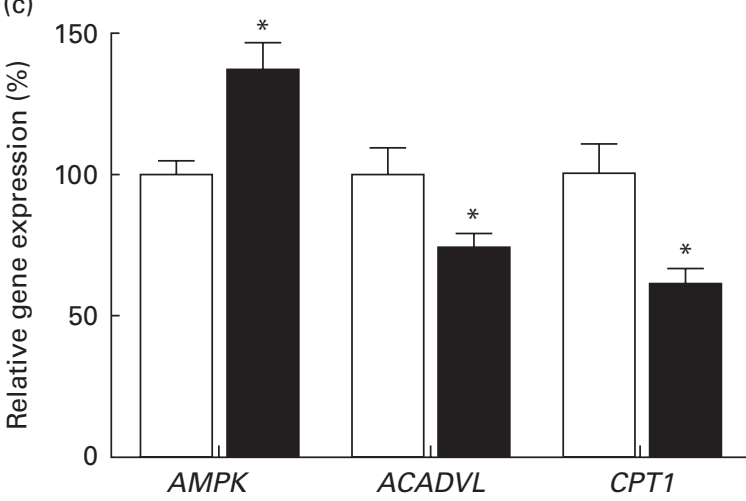

Fig. 4. Western blotting (WB) of hepatic phospho-AMP-activated protein kinase (p-AMPK)/AMPK (a) and acyl-CoA dehydrogenase, very long chain (ACADVL) (b) in the offspring of dams fed a high-fat diet (HFD-O group, $\square$ ) and standard chow (SC-O group, $\square$ ) (day 28). For the control of gel loading in WB, membranes were reblotted with $\beta$-actin. mRNA levels (quantitative real-time PCR (qRT-PCR)) of hepatic AMPK, ACADVL and carnitine palmitoyltransferase 1 (CPT1) (c). For relative gene expression analysis, glyceraldehyde-3-phosphate dehydrogenase was used as the endogenous control. Values are means ( $n 4$ for WB and $n 8$ for qRT-PCR), with their standard errors represented by vertical bars. * Mean value was significantly different from that of the SC-O group $(P \leq 0.05)$.

IкB kinase activation in association with elevated TAG content and reduced phosphorylation of AKT and ACC, limiting the steps of de novo lipid synthesis ${ }^{(6)}$. Similarly, as shown here, HFD-O mice (day 28) exhibited insulin resistance and alterations in glucose homeostasis, although ACC phosphorylation was similar in HFD-O and SC-O mice. Furthermore, although hepatic TAG and serum lipid levels were increased in abundance in HFD-O mice, the expression of enzymes involved in fatty acid biosynthesis (ACC and FAS) was not affected by maternal HFD consumption. However, HFD-O mice highly expressed SCD1, which converts stearate (18:0) to oleate (18:1) and palmitate $(16: 0)$ to palmitoleate $(16: 1)$. Previous studies have shown that inhibition of SCD1 expression by SCD1-specific antisense oligonucleotides reduced blood insulin levels, de novo fatty acid synthesis, steatosis and expression of lipogenic genes, and increased fatty acid oxidisation in primary mouse hepatocytes and the expression of genes promoting energy expenditure in the liver and adipose tissue $^{(41,42)}$. Recently, animal studies indicating a relationship between SCD1 expression and metabolic disorders have been reported ${ }^{(43)}$. Furthermore, HFD-O mice also increased
AGPAT1 expression, which catalyses the conversion of lysophosphatidate to phosphatidate, adding an acyl group to the $s n-2$ position of the glycerol backbone in the TAG synthesis pathway ${ }^{(44)}$. The role played by AGPAT1 in hepatic steatosis is still poorly understood. However, a recent study has shown that the knockdown of AGPAT1 in hepatocytes isolated from liver-specific knockout mice of the Mir122 locus reduced TAG synthesis, suggesting that AGPAT1 plays a key role in liver TAG accumulation in these mice ${ }^{(45)}$.

Increased de novo fatty acid synthesis (lipogenesis) and decreased $\beta$-oxidation activity can lead to hepatic steatosis $^{(12,13)}$. As evidence, investigators have shown that maternal consumption of a HFD affects total cholesterol and LDL-cholesterol levels and brain fatty acid composition ${ }^{(5,46)}$. In support of the literature, HFD-O mice had more levels of serum cholesterol, NEFA and TAG than SC-O mice. It is important to point out that maternal HFD consumption during lactation can contribute to milk composition ${ }^{(47,48)}$, and therefore suckling periods could affect serum lipid levels. Although HFD-O mice received the SC diet for 1 week after weaning, the impact of milk composition on the serum 


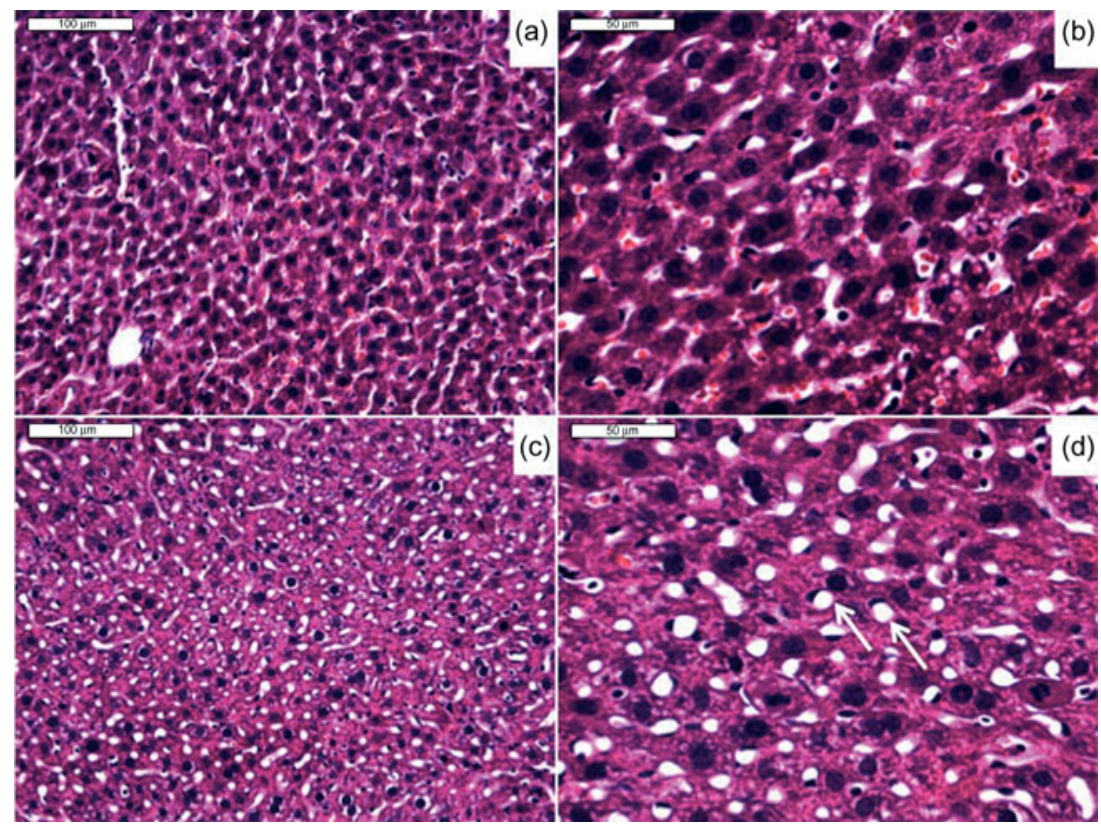

Fig. 5. Photomicrographs of the liver stained with haematoxylin-eosin from mice at day 28 showing vacuoles that contained lipids (arrows). (a, b) Offspring of dams fed a standard chow (original magnification $200 \times$ and $400 \times$, respectively, $n$ 8). (c, d) Offspring of dams fed a high-fat diet (original magnification $200 \times$ and $400 \times$, respectively, $n 8$ ).

(a)

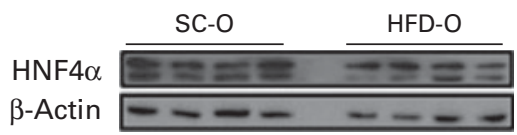

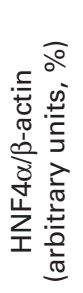

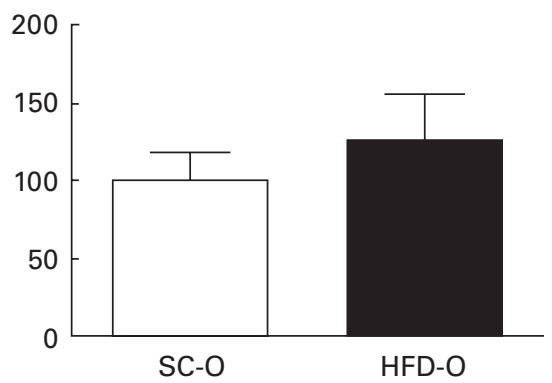

(c)
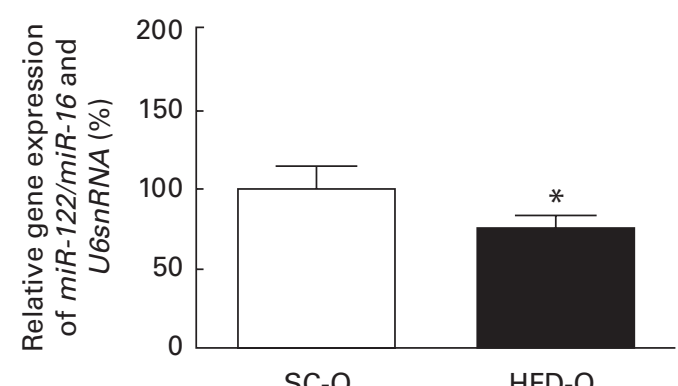

(b)

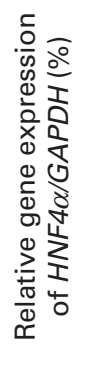

(d)
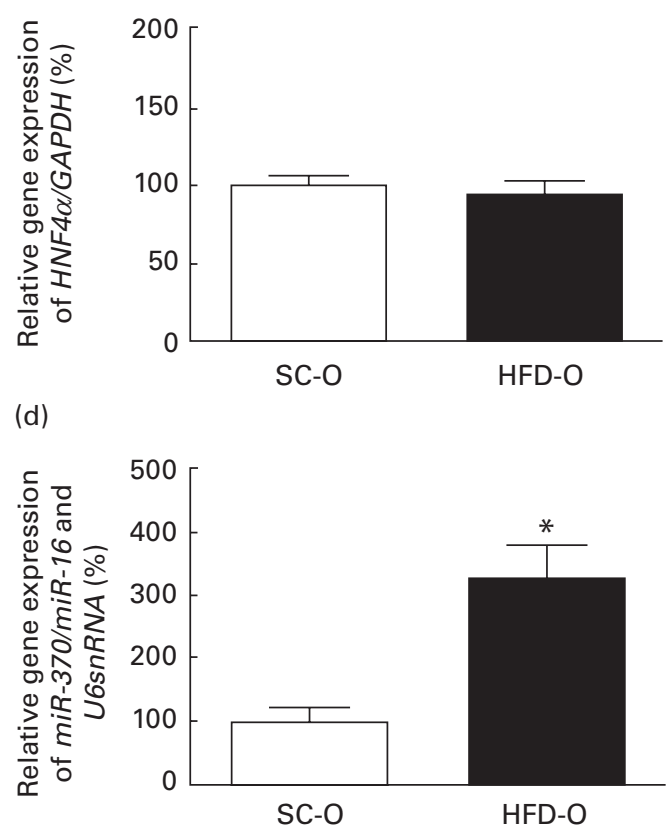

Fig. 6. Western blotting (WB) (a) and mRNA levels (b) of hepatic hepatocyte nuclear factor $4, \alpha(H N F 4 \alpha)$. MicroRNA (miRNA) levels of hepatic miRNA-122 (miR-122) (c) and miRNA-370 (miR-370) (d) in the offspring of dams fed a high-fat diet (HFD-O group) and standard chow (SC-O group) (day 28). For the control of gel loading in WB, membranes were reblotted with $\beta$-actin. For relative gene expression analysis, glyceraldehyde-3-phosphate dehydrogenase (GAPDH), miRNA-16 (miR-16) and U6 spliceosomal RNA (U6snRNA) were used as the endogenous controls. Values are means $(n 4$ for WB and $n 8-9$ for quantitative real-time PCR), with their standard errors represented by vertical bars. * Mean value was significantly different from that of the SC-O group ( $P \leq 0.05)$. 
lipid profile cannot be overlooked. In physiological conditions, potential sources of fatty acids that contribute to liver TAG deposition include hydrolysis in adipose tissue, dietary uptake and de novo lipogenesis in the liver.

Studies in human subjects and rodents have shown that excessive accumulation of liver TAG mainly results from the overflow of fatty acids generated by lipolysis in insulinresistant adipose tissue ${ }^{(49,50)}$. Donnelly et al. ${ }^{(51)}$ showed that steatosis arose from circulating fatty acids in $60 \%$ of patients and from de novo lipogenesis in $25 \%$ of patients. In agreement with the literature, the present results show that HFD-O mice have more adipose tissue and serum NEFA levels than SC-O mice. Furthermore, maternal consumption of a HFD affected SCD1 (increased expression), AGPAT1 (increased expression) and genes related to fatty acid oxidation (reduced expression of $A C A D V L$ and CPT1), but did not affect enzymes important for fatty acid synthesis. Altogether, these results suggest that hepatic TAG accumulation in HFD-O mice can also be due to diminished fatty acid oxidation. Moreover, the high SCD1 enzyme activity could lead to increased availability of MUFA for TAG synthesis in HFD-O mice, and the increase in AGPAT1 expression in HFD-O mice reinforces this hypothesis.

Many studies have indicated the importance of miRNA in lipid metabolism and liver physiology and disease ${ }^{(19,52-56)}$, but the role of these small non-coding RNA in hepatic lipid metabolism is still controversial. Esau et al. ${ }^{(52)}$ showed that inhibition of miR-122 expression reduced fatty acid synthesis and increased fatty acid oxidation. Recently, two studies ${ }^{(45,57)}$ employing genetic deletion in mice have provided substantial evidence of miR-122 function in lipid metabolism. Hsu et $a l .{ }^{(45)}$ showed the up-regulation of genes involved in lipid synthesis in miR-deficient liver such as AGPAT, phosphatidic acid phosphatase type 2A (Ppap2a) and monoacylglycerol transferases (Mogat). Consistent with the changes in gene expression, the mutant liver synthesised more, but secreted less, TAG than the control liver, resulting in TAG accumulation in mutant hepatocytes. In addition, both groups also showed an increase in the number of infiltrating inflammatory cells in the liver of miR-122-deficient mice ${ }^{(45,57)}$, suggesting a proinflammatory effect of reduced expression of this miRNA. Interestingly, HFD-O mice exhibited reduced expression of $m i R-122$, increased liver TAG deposition and JNK activation, although SCD1 increased in expression. Others have shown that suppression of $m i R-122$ in non-human primates reduces plasma cholesterol ${ }^{(58,59)}$. In the present study, HFD-O mice expressed less HMGCR than SC-O mice, but the level of plasma cholesterol were increased rather than reduced. It is possible that maternal consumption of a HFD modifies milk composition and affects the cholesterol level in plasma. We also found that HFD-O mice expressed more $m i R-370$ in the liver than SC-O mice. This miRNA controls the expression of $m i R-122$ and affects lipid metabolism ${ }^{(19)}$. miR-370 activates lipogenic genes indirectly through $m i R-122$ in a different experimental model. Importantly, miR-370 directly downregulates $\mathrm{CPT} 1 \alpha$, which controls the rate-limiting step in fatty acid $\beta$-oxidation. In agreement with the literature, HFD-O mice also had reduced expression of CPT1 $\alpha$ and possibly reduced fatty acid $\beta$-oxidation, as indicated by the diminished expression level of ACADVL protein and mRNA.

Thus, hepatic lipid accumulation in the offspring of obese dams has multifactorial characteristics, and changes in the oxidative pathway are associated with the differential expression of $m i R-370$ and $m i R-122$ in the liver. In addition, HFD-O mice also had increased liver inflammation, probably because of the uterine environment. This adaptive response leads to hepatic metabolic changes that cause liver injury.

\section{Acknowledgements}

The present study was supported by São Paulo Research Foundation (FAPESP) (grant no. 2011/22156-7) and the National Council for Scientific and Technological Development (CNPq, grant no. 479017/2011-5). The funders had no direct influence over the design, conduct or reporting of the present study.

R. O. B. was supported by São Paulo Research Foundation (FAPESP) Fellow Masters in nutrition science, sport and metabolism (grant no. 2011/13947-0).

R. O. B. contributed to the development of the study hypothesis, reviewed the literature, conducted the statistical analysis, provided interpretation of the results, and drafted and edited the manuscript. A. M. M., F. O. B. and L. A. P. S. contributed to the development of the study hypothesis and provided advice regarding interpretation of the results. L. M. I.-S., M. M. and L. A. V. assisted with drafting and editing of the manuscript. M. A. T. provided advice regarding interpretation of the results and edited the manuscript. A. S. T. was responsible for the study design, data collection, provided advice regarding interpretation of the results and edited the manuscript.

None of the authors has any conflict of interest to declare.

\section{References}

1. Danielzik S, Langnäse K, Mast M, et al. (2002) Impact of parental BMI on the manifestation of overweight 5-7 year old children. Eur J Nutr 41, 132-138.

2. Boney CM, Verma A, Tucker R, et al. (2005) Metabolic syndrome in childhood: association with birth weight, maternal obesity, and gestational diabetes mellitus. Pediatrics $\mathbf{1 1 5}$, e290-e296.

3. Catalano PM, Farrell K, Thomas A, et al. (2009) Perinatal risk factors for childhood obesity and metabolic dysregulation. Am J Clin Nutr 90, 1303-1313.

4. Challier JC, Basu S, Bintein T, et al. (2008) Obesity in pregnancy stimulates macrophage accumulation and inflammation in the placenta. Placenta 29, 274-281.

5. Elahi MM, Cagampang FR, Mukhtar D, et al. (2009) Longterm maternal high-fat feeding from weaning through pregnancy and lactation predisposes offspring to hypertension, raised plasma lipids and fatty liver in mice. Br J Nutr $\mathbf{1 0 2}$, 514-519.

6. Ashino NG, Saito KN, Souza FD, et al. (2012) Maternal highfat feeding through pregnancy and lactation predisposes mouse offspring to molecular insulin resistance and fatty liver. J Nutr Biochem 23, 341-348.

7. Shankar K, Zhong Y, Kang P, et al. (2011) Maternal obesity promotes a proinflammatory signature in rat uterus and blastocyst. Endocrinology 152, 4158-4170. 
8. Qureshi K \& Abrams GA (2007) Metabolic liver disease of obesity and role of adipose tissue in the pathogenesis of nonalcoholic fatty liver disease. World $J$ Gastroenterol 13, $3540-3553$.

9. Stanton MC, Chen SC, Jackson JV, et al. (2011) Inflammatory signals shift from adipose to liver during high fat feeding and influence the development of steatohepatitis in mice. $J$ Inflamm (Lond) $\mathbf{8}, 8$.

10. McCurdy CE, Bishop JM, Williams SM, et al. (2009) Maternal high-fat diet triggers lipotoxicity in the fetal livers of nonhuman primates. J Clin Invest 119, 323-335.

11. Heerwagen MJ, Stewart MS, de la Houssaye BA, et al. (2013) Transgenic increase in $n-3 / n-6$ fatty acid ratio reduces maternal obesity-associated inflammation and limits adverse developmental programming in mice. PLOS ONE 8, e67791.

12. Postic C \& Girard J (2008) The role of the lipogenic pathway in the development of hepatic steatosis. Diabetes Metab 34, 643-648.

13. Bouanane S, Merzouk H, Benkalfat NB, et al. (2010) Hepatic and very low-density lipoprotein fatty acids in obese offspring of overfed dams. Metabolism 59, 1701-1709.

14. Waterland RA (2005) Does nutrition during infancy and early childhood contribute to later obesity via metabolic imprinting of epigenetic gene regulatory mechanisms? Nestle Nutr Workshop Ser Pediatr Program 56, 157-171, discussion 171-154.

15. Sullivan EL \& Grove KL (2010) Metabolic imprinting in obesity. Forum Nutr 63, 186-194.

16. Bartel DP (2004) MicroRNAs: genomics, biogenesis, mechanism, and function. Cell 116, 281-297.

17. Li ZY, Xi Y, Zhu WN, et al. (2011) Positive regulation of

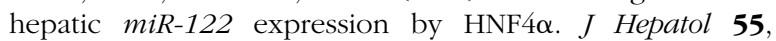
602-611.

18. Yang YM, Seo SY, Kim TH, et al. (2012) Decrease of microRNA-122 causes hepatic insulin resistance by inducing protein tyrosine phosphatase $1 \mathrm{~B}$, which is reversed by licorice flavonoid. Hepatology 56, 2209-2220.

19. Iliopoulos D, Drosatos K, Hiyama Y, et al. (2010) MicroRNA370 controls the expression of microRNA-122 and Cpt1 $\alpha$ and affects lipid metabolism. J Lipid Res 51, 1513-1523.

20. Dell RB, Holleran S \& Ramakrishnan R (2002) Sample size determination. ILAR J 43, 207-213.

21. Carr TP, Andresen CJ \& Rudel LL (1993) Enzymatic determination of triglyceride, free cholesterol, and total cholesterol in tissue lipid extracts. Clin Biochem 26, 39-42.

22. Newberry EP, Xie Y, Kennedy S, et al. (2003) Decreased hepatic triglyceride accumulation and altered fatty acid uptake in mice with deletion of the liver fatty acid-binding protein gene. J Biol Chem 278, 51664-51672.

23. Bonora E, Zavaroni I, Alpi O, et al. (1987) Relationship between blood pressure and plasma insulin in non-obese and obese non-diabetic subjects. Diabetologia 30, 719-723.

24. Stenseth NC, Viljugrein H, Saitoh T, et al. (2003) Seasonality, density dependence, and population cycles in Hokkaido voles. Proc Natl Acad Sci US A 100, 11478-11483.

25. Shoelson SE, Lee J \& Yuan M (2003) Inflammation and the IKK beta/I kappa B/NF-kappa B axis in obesity- and dietinduced insulin resistance. Int J Obes Relat Metab Disord 27, Suppl. 3, S49-S52.

26. Cnop M (2008) Fatty acids and glucolipotoxicity in the pathogenesis of type 2 diabetes. Biochem Soc Trans 36, 348-352.

27. Araújo EP, Torsoni MA \& Velloso LA (2010) Hypothalamic inflammation and obesity. Vitam Horm 82, 129-143.

28. Donath MY \& Shoelson SE (2011) Type 2 diabetes as an inflammatory disease. Nat Rev Immunol 11, 98-107.

29. Gregor MF \& Hotamisligil GS (2011) Inflammatory mechanisms in obesity. Annu Rev Immunol 29, 415-445.
30. Horng T \& Hotamisligil GS (2011) Linking the inflammasome to obesity-related disease. Nat Med 17, 164-165.

31. Velloso LA \& Schwartz MW (2011) Altered hypothalamic function in diet-induced obesity. Int $J$ Obes (Lond) 35 $1455-1465$.

32. Levin BE (2010) Interaction of perinatal and pre-pubertal factors with genetic predisposition in the development of neural pathways involved in the regulation of energy homeostasis. Brain Res 1350, 10-17.

33. Barbuio R, Milanski M, Bertolo MB, et al. (2007) Infliximab reverses steatosis and improves insulin signal transduction in liver of rats fed a high-fat diet. J Endocrinol 194, 539-550.

34. Cintra DE, Pauli JR, Araújo EP, et al. (2008) Interleukin-10 is a protective factor against diet-induced insulin resistance in liver. J Hepatol 48, 628-637.

35. Wiedemann MS, Wueest S, Item F, et al. (2013) Adipose tissue inflammation contributes to short-term high-fat dietinduced hepatic insulin resistance. Am J Physiol Endocrinol Metab 305, E388-E395.

36. Turner N, Kowalski GM, Leslie SJ, et al. (2013) Distinct patterns of tissue-specific lipid accumulation during the induction of insulin resistance in mice by high-fat feeding. Diabetologia 56, 1638-1648.

37. Kraegen EW \& Cooney GJ (2008) Free fatty acids and skeletal muscle insulin resistance. Curr Opin Lipidol 19, 235-241.

38. Samuel VT \& Shulman GI (2012) Mechanisms for insulin resistance: common threads and missing links. Cell 148, 852-871.

39. Farese RV Jr, Zechner R, Newgard CB, et al. (2012) The problem of establishing relationships between hepatic steatosis and hepatic insulin resistance. Cell Metab 15, 570-573.

40. Brown MS \& Goldstein JL (2008) Selective versus total insulin resistance: a pathogenic paradox. Cell Metab 7, 95-96.

41. Jiang G, Li Z, Liu F, et al. (2005) Prevention of obesity in mice by antisense oligonucleotide inhibitors of stearoyl-CoA desaturase-1. J Clin Invest 115, 1030-1038.

42. Yokoyama S, Hosoi T \& Ozawa K (2012) Stearoyl-CoA desaturase 1 (SCD1) is a key factor mediating diabetes in MyD88-deficient mice. Gene 497, 340-343.

43. Hodson L \& Fielding BA (2013) Stearoyl-CoA desaturase: rogue or innocent bystander? Prog Lipid Res 52, 15-42.

44. Takeuchi K \& Reue K (2009) Biochemistry, physiology, and genetics of GPAT, AGPAT, and lipin enzymes in triglyceride synthesis. Am $J$ Physiol Endocrinol Metab 296, E1195-E1209.

45. Hsu SH, Wang B, Kota J, et al. (2012) Essential metabolic, anti-inflammatory, and anti-tumorigenic functions of miR-122 in liver. J Clin Invest 122, 2871-2883.

46. Yu H, Bi Y, Ma W, et al. (2010) Long-term effects of high lipid and high energy diet on serum lipid, brain fatty acid composition, and memory and learning ability in mice. Int J Dev Neurosci 28, 271-276.

47. Silber GH, Hachey DL, Schanler RJ, et al. (1988) Manipulation of maternal diet to alter fatty acid composition of human milk intended for premature infants. Am J Clin Nutr 47, 810-814.

48. Priego T, Sánchez J, García AP, et al. (2013) Maternal dietary fat affects milk fatty acid profile and impacts on weight gain and thermogenic capacity of suckling rats. Lipids $\mathbf{4 8}$, 481-495.

49. Lewis GF, Carpentier A, Adeli K, et al. (2002) Disordered fat storage and mobilization in the pathogenesis of insulin resistance and type 2 diabetes. Endocr Rev 23, 201-229.

50. Ferré P \& Foufelle F (2010) Hepatic steatosis: a role for de novo lipogenesis and the transcription factor SREBP-1c. Diabetes Obes Metab 12, Suppl. 2, 83-92. 
51. Donnelly KL, Smith CI, Schwarzenberg SJ, et al. (2005) Sources of fatty acids stored in liver and secreted via lipoproteins in patients with nonalcoholic fatty liver disease. J Clin Invest 115, 1343-1351.

52. Esau C, Davis S, Murray SF, et al. (2006) miR-122 regulation of lipid metabolism revealed by in vivo antisense targeting. Cell Metab 3, 87-98.

53. Li S, Chen X, Zhang H, et al. (2009) Differential expression of microRNAs in mouse liver under aberrant energy metabolic status. J Lipid Res 50, 1756-1765.

54. Whittaker R, Loy PA, Sisman E, et al. (2010) Identification of microRNAs that control lipid droplet formation and growth in hepatocytes via high-content screening. J Biomol Screen 15, 798-805.
55. Rayner KJ, Sheedy FJ, Esau CC, et al. (2011) Antagonism of miR-33 in mice promotes reverse cholesterol transport and regression of atherosclerosis. J Clin Invest 121, 2921-2931.

56. Aranda JF, Madrigal-Matute J, Rotllan N, et al. (2013) MicroRNA modulation of lipid metabolism and oxidative stress in cardiometabolic diseases. Free Radic Biol Med 64, 31-39.

57. Tsai WC, Hsu SD, Hsu CS, et al. (2012) MicroRNA-122 plays a critical role in liver homeostasis and hepatocarcinogenesis. J Clin Invest 122, 2884-2897.

58. Krützfeldt J, Rajewsky N, Braich R, et al. (2005) Silencing of microRNAs in vivo with 'antagomirs'. Nature 438, 685-689.

59. Elmén J, Lindow M, Schütz S, et al. (2008) LNA-mediated microRNA silencing in non-human primates. Nature 452, 896-899. 